\title{
Caracterização clínico-epidemiológica dos pacientes submetidos a implantação de dispositivos eletrônicos cardíacos em um hospital terciário privado Angolano
}

Miguel Bernardino Antunes Vicente 1, José Ribeiro Bunda Ricardo 1, Domingas Baião 1, Edna Mahela Magalhães dos Reis Manuel 1, Telmo Martins 1, Lemuel Bornelli Cordeiro 1, Pedro Sabola 1, António Pedro Filipe Júnior 1

${ }^{1}$ Unidade de Diagnóstico e Intervenção Cardiovascular, Clínica Girassol, Luanda, Lda, Angola.

* Corresponding author: Miguel Bernardino Antunes Vicente. Street: Comandante Gika, n²25 - Maianga. Zip Code: 12345-123 - Luanda, Lda, Angola. Phone: +244 940980920. E-mail: miguelantunesv@gmail.com.

Research Ethics Committee Approval (if necessary): Aprovado pelo Gabinete de Ensino Pós-Graduação e Pesquisa da Clínica Girassol (\#08/CG/GEPP/2021).

Received on: Aug 4, 2021. Accepted on: Aug 13, 2021. Available online: Aug 25, 2021.

\section{Abstract}

Os Dispositivos Eletrônicos Cardíaco Implantáveis (DECI) são grandes aliados no tratamento de distúrbios do ritmo cardíaco, não obstante a isso, vem sofrendo alterações para ampliar as indicações em contextos variáveis como na disfunção sistólica ventricular. Este estudo objetiva caracterizar clínico-epidemiológico os pacientes submetidos a implantação de dispositivos eletrônicos cardíaco no período de 2015 a 2018, realizado através de estudo descritivo, transversal, quantitativo de carácter retrospectivo. No total foram 133 registros de pacientes submetidos a implantação desses dispositivos.

Keywords: Dispositivos Eletrônicos Cardíaco Implantáveis; Arritmias; Disfunção sistólica ventricular.

\section{Introdução}

As Doenças Cardiovasculares (DCV) incluindo as arritmias cardíacas, se mantêm como as principais causas de mortes em todo mundo há mais duas décadas. Em 2016 ocorreram cerca de 56,9 milhões de mortes, e 17,9 milhões foram por DCV, e infelizmente mais de
80\% dessas mortes ocorreram em países de baixa e média renda [1]. A falta de acesso ao tratamento com DECI é dentre outros fatores, responsáveis por dizimar cerca de dois milhões de pessoas por ano no mundo [2].

Desde a implementação dos DECI na prática médica, tem havido uma redução no número de mortes 
relacionadas com as arritmias e transtorno de condução do impulso cardíaco [3], não obstante a isso, vem sofrendo alterações para ampliar as indicações e atender as necessidades de cada realidade arritmogênica, garantindo aos pacientes aumento da qualidade e expectativa de vida [4-5].

No continente africano essa realidade representa um importante problema de saúde pública, principalmente em países de baixa e média renda aos quais pertencem a maioria dos países da África subsaariana, fato agravado pela falta de infra-estrutura e recursos humanos disponíveis, alto custo dos procedimentos, condições sociais desfavoráveis, que contribuem para manter a baixa acessibilidade dos mesmos [6].

As arritmias cardíacas em sua maioria subdiagnosticadas e os procedimentos de estimulação pouco desenvolvidos contribuem para $\mathrm{O}$ manuseamento débil no nosso meio [68], com realce na taxa de implantação desses dispositivos, menor em 200 vezes em relação a Europa Ocidental [9]. Cenário preocupante, uma vez que, este tipo de tratamento está associado à redução substancial da morbimortalidade por transtornos do ritmo e condução do impulso nos países industrializados [2]. Esse estudo teve como objetivo caracterizar clínicoepidemiológico os pacientes submetidos a implantação de Dispositivo eletrônico Cardíaco (DEC) no período de janeiro de 2015 a dezembro de 2018 em um hospital terciário privado na cidade de Luanda.

\section{Metodologia}

Trata-se de um estudo descritivo, transversal quantitativo e de carácter retrospectivo, realizado na Clínica Girassol, envolvendo 133 pacientes, dos quais foram implantados um DEC. Os dados foram coletados na base de dados do Centro Cardiovascular e Torácico. O período de estudo foi marcado entre janeiro 2015 a dezembro 2018.

As variáveis utilizadas para análise foram: sexo, idade, indicação clínica para implantação, modo de estimulação, convênio de saúde e principais complicações. Os resultados foram apresentados sob a forma de gráficos e tabelas em valores absolutos e percentuais. A análise foi realizada utilizando o software Statistical Package for Social Science (SPSS) 12.0.

As variáveis categóricas foram expressas com frequências absolutas e relativas, as contínuas como média \pm desvio padrão ou mediana e percentis $25 \%$ e $75 \%$, conforme seguissem ou não distribuição normal. As variáveis categóricas foram comparadas entre grupos usando o teste do Qui-quadrado, e calculados os intervalos de confiança de $95 \%$ para as prevalências. Foram considerados significativos valores de $\mathrm{p}<0,05$.

\section{Resultados}

O presente estudo incluiu 133 pacientes de ambos sexos submetidos a 
implantação de DEC. A média de idade foi de $58 \pm 10,5$ anos. A tabela 1 ilustra a distribuição dos pacientes de acordo ao gênero e faixa etária, onde houve predomínio dos indivíduos do sexo masculino entre os 20 e 80 anos. $\mathrm{O}$ sexo feminino foi predominante nos pacientes maiores de 80 anos.

Tabela 1. Distribuição dos pacientes de acordo ao gênero e grupo e faixa etária.

\begin{tabular}{ccccc}
\hline \multirow{2}{*}{$\begin{array}{c}\text { Grupo de } \\
\text { idade }\end{array}$} & \multicolumn{2}{c}{ Gênero } & \multirow{2}{*}{ Total n (\%) } & $p$ \\
\cline { 2 - 3 } $20-40$ & $2(1,5)$ & $5(3,8)$ & $7(5,3)$ & 0,002 \\
$41-60$ & $7(5,3)$ & $19(14,3)$ & $26(19,5)$ & \\
$61-80$ & $34(25,6)$ & $37(27,8)$ & $71(53,4)$ & \\
$>80$ & $22(16,5)$ & $7(5,3)$ & $29(21,8)$ & \\
Total & $65(48,9)$ & $68(51,1)$ & $133(100)$ & \\
\hline
\end{tabular}

Legenda: A percentagem foi calculada a partir do total de pacientes $(n=133)$. Como prova estatística utilizou-se a prova $X^{2}$. Foram considerados significativos os valores de $\mathrm{p}<0,05$.

Na Tabela 2, são apresentados os diferentes fatores de risco presentes nos pacientes estudados segundo o sexo. A Hipertensão Arterial e a Dislipidemia foram os principais fatores de risco associados, $\quad(80,5 \%$ e $\quad 33,8 \%$, respetivamente); sem significância estatística quando comparados homens e mulheres em relação aos mesmos. Somente $18,8 \%$ da amostra eram diabéticos.

Tabela 2. Distribuição dos pacientes de acordo aos fatores de risco.

\begin{tabular}{|c|c|c|c|c|}
\hline \multirow[t]{2}{*}{ Fatores de risco } & \multicolumn{2}{|c|}{ Gênero } & \multirow{2}{*}{$\begin{array}{l}\text { Total } \\
\text { n (\%) }\end{array}$} & \multirow{2}{*}{$p$} \\
\hline & Feminino n (\%) & Masculino n (\%) & & \\
\hline HTA & $51(47,7)$ & $56(52,3)$ & $107(80,5)$ & 0,73 \\
\hline $\mathrm{DM}_{2}$ & $16(64,0)$ & $9(36,0)$ & $25(18,8)$ & 0,14 \\
\hline Dislipidemia & $20(44,4)$ & $25(55,6)$ & $45(33,8)$ & 0,58 \\
\hline Tabagismo & $16(57,1)$ & $12(42,9)$ & $28(21,1)$ & 0,44 \\
\hline
\end{tabular}

Legenda: HTA: Hipertensão Arterial; DM2: Diabetes Mellitus tipo 2. Nota esclarecedora: A percentagem foi calculada a partir do total de pacientes $(n=133)$. Como prova estatística utilizou-se a prova $\mathrm{X}^{2}$ com correção para (HTA, DM, Dislipidemia e o tabagismo). Foram considerados significativos os valores de $\mathrm{p}<0,05$. 
O gráfico 1, ilustra a indicação clínica mais frequente para a implantação de DEC, onde o Bloqueio atrioventricular total (BAVT) foi a arritmia mais frequentemente documentada (46\%), seguido da Doença do nó sinusal (22\%). O bloqueio atrioventricular $2^{\circ}$ Mobitz 2 e a Fibrilação atrial com resposta ventricular lenta representaram (13 e $9 \%$, respectivamente). O esgotamento das baterias culminou em reoperações para substituição do gerador em $9 \%$ dos casos.

Gráfico 1. Distribuição dos pacientes submetidos a implantação de DEC de acordo a indicação clínica, 2011 - 2018.

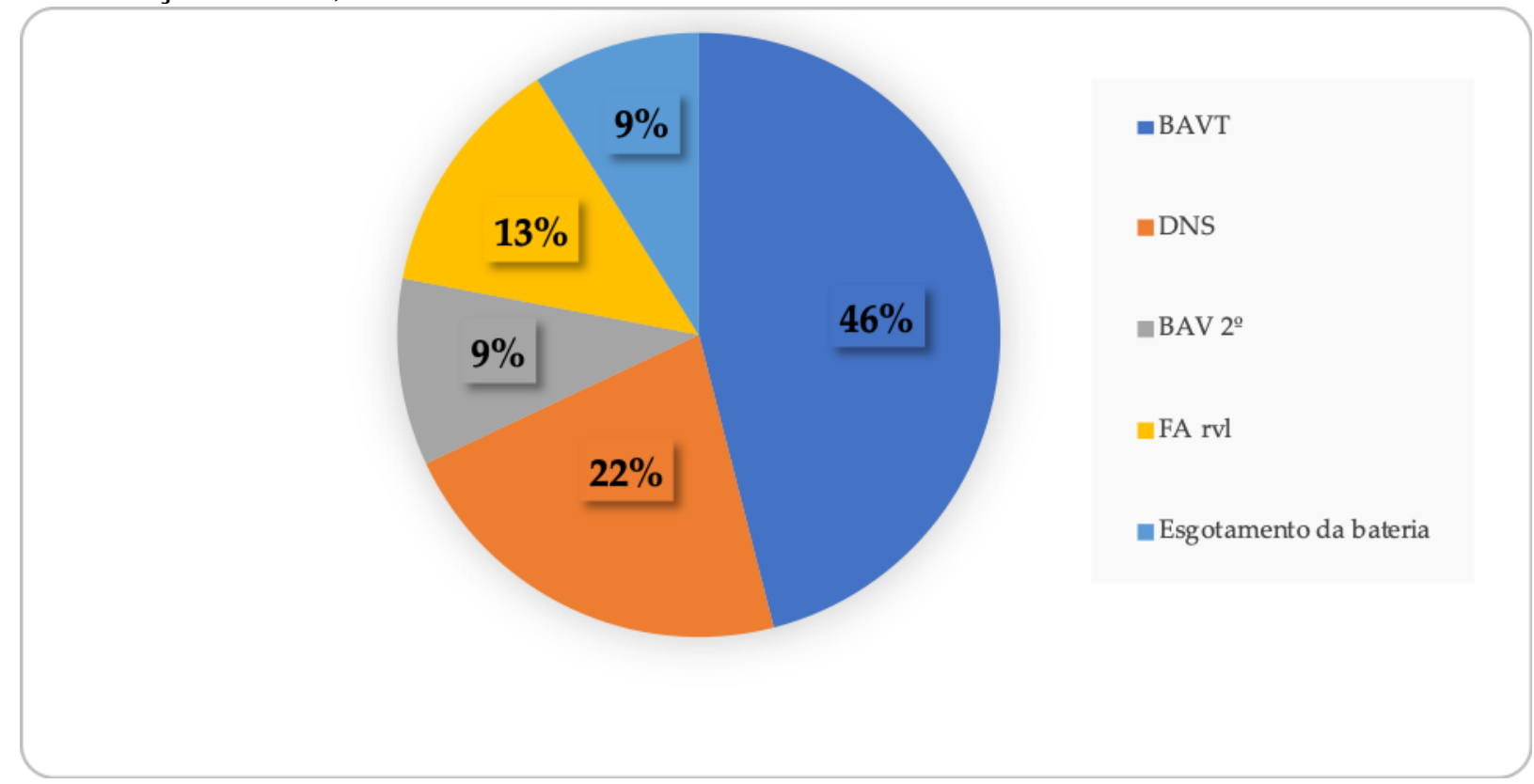

Legenda: BAVT: Bloqueio atrioventricular total; DNS: Doença do nó sinusal;

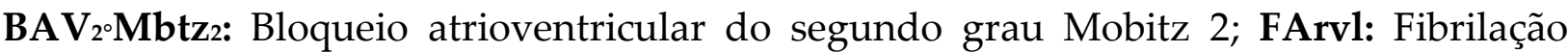
atrial com resposta ventricular lenta.

$\mathrm{Na}$ distribuição dos pacientes de acordo ao modo de estimulação cardíaca utilizada, a estimulação bicameral foi a mais utilizada em 52,6\%, seguido de estimulação unicameral 41,3\%. Os Cardio-desfibriladores implantáveis (CDI) e Terapia de cardioressincronização e desfibrilação (CRT-D) apresentaram baixas taxas de implantações $\quad(0,8 \%$ e $\quad 1,5 \%$ respetivamente) (Tabela 3). A seguir está ilustrado a modalidade de pagamento para ter acesso as implantações desses DEC, a maioria destas foram custeadas através de um convênio de saúde $(81,2 \%)$, conforme a tabela 4 .

As principais eventualidades a curto prazo relacionadas com o processo 
de implantação estão refletidas na tabela 5. A maioria dos procedimentos ocorreu sem complicações $(84,5 \%)$. A infecção da loca do DEC constituiu a complicação mais frequente em $7,1 \%$ dos casos, seguido do deslocamento do eletrocateter em $5,2 \%$ e o hematoma/equimose da região do implante $3,2 \%$.

Tabela 3. Distribuição dos pacientes submetidos a implantação de DEC de acordo ao modo de estimulação cardíaca utilizado.

\begin{tabular}{cc}
\hline Modo de estimulação & $\mathbf{n}(\mathbf{\%})$ \\
\hline VVIR & $55(41,3)$ \\
VDD & $5(3,8)$ \\
DDDR & $70(52,6)$ \\
CDI & $1(0,8)$ \\
CRT-D & $2(1,5)$ \\
Total & $133(100)$ \\
\hline
\end{tabular}

Legenda: VVI: Marcapasso de câmara única; VDD/DDD: marcapasso de dupla câmara; CRT-D: Terapia de cardio-ressincronização e desfibrilação; CDI: Cardiodesfibrilador Implantável.

Tabela 4. Distribuição dos pacientes submetidos a implantação de DEC de acordo ao convênio, 2011 - 2018.

\begin{tabular}{cr}
\hline Convênio & n (\%) \\
\hline Particular & $25(18,8)$ \\
Seguro de saúde & $108(81,2)$ \\
Total & $133(100)$ \\
\hline
\end{tabular}

Tabela 5. Distribuição dos pacientes submetidos a implantação de DEC segundo as complicações mais frequentes, 2011 - 2018.

\begin{tabular}{cc}
\hline Complicações & $\mathbf{n}(\mathbf{\%})$ \\
\hline Infecções & $9(7,1)$ \\
Hematoma / Equimose & $4(3,2)$ \\
Deslocamento do eletrocateter & $7(5,2)$
\end{tabular}




\section{Discussão e Conclusão}

Angola é um país com 18 províncias, situado na parte ocidental do continente africano precisamente na zona Austral. Com uma extensão territorial de cerca de $1.246 .700 \mathrm{~km}^{2}$, onde o crescimento populacional tende a aumentar para 34 milhões de habitantes até o final de 2022 [10].

O país conta com 4 centros de implantações de DEC (2 públicos e 2 privados), todos eles concentrados numa única província (a capital Luanda), onde somente os privados realizam implantações atualmente, e por conta dos altos custos desses procedimentos, milhares de pacientes deixam de ter o tratamento considerado padrão no mundo inteiro, aumentando a grande carga de pacientes em lista de espera na rede pública, que muitas vezes por opção individual recorrem a outros países como a África do Sul, Portugal e Brasil, onde há maior disponibilidade dos mesmos e custos mais atrativos.

Essa realidade de pouco desenvolvimento da atividade de estimulação cardíaca em Angola por razões econômicas, déficit de centros, recursos materiais e humanos especializados torna encarecido $\mathrm{O}$ tratamento e beneficia uma pequena parcela de pacientes (aqueles com capacidade financeira para arcar com os altos custos, os pacientes com seguro de saúde e aqueles que podem realizar no exterior), deixando o país abaixo das 10 implantações por milhão de habitantes e sem poder oferecer a maioria dos pacientes esse tratamento específico.

Este é o primeiro estudo angolano que caracterizou clínicoepidemiológico os pacientes submetidos implantação de dispositivos eletrônicos cardíaco. Num período de Janeiro de 2015 a Dezembro de 2018 na nossa unidade hospitalar, houve predomínio da raça negra como esperado pelo contexto local da investigação, o sexo masculino representou a maioria com uma média de idade $58 \pm 10,5$ anos.

Esses dados coincidem com os resultados de estudos realizados na Nigéria e Burkina Faso no período de 5 anos, onde o sexo masculino foi o mais prevalente e a idade média variou entre 68-69 anos [11,12], mostrando que esse gênero vem sendo cada vez mais acometido por distúrbios de ritmo. Chama a atenção ainda que houve um predomínio de mulheres acima de 80 anos, com manifestações tardias em relação aos homens, e que pode ser explicado pelo aumento de prevalência de DCV no período pós-menopausa onde há declinação da função ovárica com consequente degeneração das estruturas cardíacas em especial o sistema de condução de impulsos [12].

Vários fatores estão implicados no aumento da incidência de HTA entre os angolanos, desde os fatores não modificáveis a questões relacionadas a adoção de novos estilos de vida, por conta das mudanças econômicas, industrialização, rápida urbanização, o que vem conduzindo a população a uma célere transição epidemiológica 
aumentando gradualmente a prevalência de HTA principalmente em indivíduos do sexo masculino e de menor estrato socioeconômico [13].

No estudo em causa, a HTA foi o principal fator de risco e mais prevalente no sexo masculino $(52,3 \%)$.

Alguns países africanos $[6,11,14,15]$ realizaram forças tarefas no intuito de conhecer o perfil clínico-epidemiológico dos pacientes submetidos a implantação de DEC, e os resultados foram consonantes em identificar o BAVT como o distúrbio de ritmo mais frequente entre os pacientes estudados que motivou a implantação desses dispositivos. No estudo em questão não foi diferente em ratificar este achado. Apesar da semelhança dos dados, vale realçar que há necessidade de criação de uma base de dados nacional em Angola para melhor conhecer esse cenário que motiva a implantação de DEC.

A seleção do modo de estimulação ótima é vital para oferecer uma capacidade funcional de acordo a necessidade fisiológica dos pacientes, não obstante a isso, algoritmos de programação dos dispositivos, vêm sendo melhorado nas últimas décadas. $\mathrm{Na}$ presente investigação, o modo de estimulação bicameral foi o mais utilizado, escolha comum em centros mais experientes, evidentemente individualizando o contexto de cada paciente [15], mostrando uma tendência de maior eficácia em comparação com a câmera única [16]. Essa facilidade de seleção de DECI para atender a cada especificidade de arritmia em muitos países africanos torna-se um desafio frente a pouca disponibilidade dos mesmos, sobretudo em países de baixa renda $[17,18]$.

O melhor manuseamento desses DECI assim como o preparo pré, trans e pós-implantação deve ser realizado no intuito de reduzir o máximo a probabilidade de infecções relacionado com o procedimento. Neste estudo a infecção do local de implantação do dispositivo e o deslocamento de eletrocateteres foram as complicações mais frequentes, resultado coincidente com o realizado na Nigéria durante 5 anos em pacientes submetidos a implantações de DEC [18]. Na nossa instituição esse número tende a reduzir por conta da criação de protocolos de assistência do paciente envolvido, utilizando a antibioticoterapia profilática e outros cuidados gerais [5].

$\mathrm{O}$ estudo mostrou ainda que as implantações desses DEC foram realizadas maioritariamente em pacientes que possuíam um convênio de saúde, fato que permitiu acesso integral ao tratamento, por outro lado, embora haja grande número de pacientes que se beneficiariam desse tratamento, a falta de apoio financeiro foi a principal razão para baixa acessibilidade entre os mesmos, uma vez que em sua maioria foram realizados em centros privados, realidade que coincide com outros autores africanos [2,6,11,19], que revelaram que a principal razão para baixo acesso foi a falta de condições financeiras para arcar com os altos custos do procedimento.

Provavelmente seguindo a experiência estratégica de outros países como Gana e Nigéria com a reutilização desses DECI post-mortem [2-3] 
provenientes de países europeus e não só, melhoraria a acessibilidade desses pacientes, pois é uma iniciativa que salva vidas em países de baixa e média renda e que de fato pode ser uma alternativa benéfica para Angola. Embora seja controverso, pelo receio sobre a taxa de infecções relacionada a reutilização desses dispositivos, a mesma é assegurada por uma esterilização que segue normas rígidas de segurança fazendo com que essa taxa de infecção não tenha diferença significativa em relação aos dispositivos novos [3].

O certo é que em meio a essa situação de gestão para melhorar a acessibilidade dos pacientes com distúrbios de condução ao tratamento ideal, a implantação dos DEC já é uma realidade em Angola porém, ainda está distante de alcançar a maioria da população que necessita desse tratamento, entretanto precisa-se urgentemente de serem criadas estratégias para melhorar o acesso desses pacientes.

\section{Referências}

[1] World Health Organization (WHO). Cardiovascular Diseases. Fact sheet N.317. Updated March 2013. Geneva: WHO. Disponível em: https:/www.who.int/news-room/factsheets/detail/cardiovascular-diseases(cvds).

[2] Sani MU, Mayosi BM. The Pacemaker and ICD Reuse Programme of the Pan-African Society of Cardiology. Heart. 2017
Dec;103(23):1844-1845.

doi: 10.1136/heartjnl-2017-311462.

[3] Tandon K, Tate T, Kirkpatrick JN. Pacemaker reuse in low-income/middleincome countries: moral duty or dangerous precedent? Heart. 2017 Dec;103(23):1846-1847. doi: 10.1136/heartjnl-2017-311572.

[4] Rapsang AG, Bhattacharyya P. Pacemakers and implantable cardioverter defibrillators-general and anesthetic considerations. Revista Brasileira de Anestesiologia. 2014 Jun;64(3):205-14.

doi:10.1016/j.bjane.2013.02.005.

[5] Ramos G, Ramos FJ, Rassi JA, Pereira E, Gabriel NS, Chaves E. Artificial cardiac pacemaker: pre and intraoperative considerations. Revista brasileira de anestesiologia. 2003 Dec;53(6):854-62. doi: 10.1590/S003470942003000600015 .

[6] Ikama SM, Makani J, Jouven X, Kimbally-Kaky G. Permanent cardiac pacing: first Congolese experiment. Pan Afr Med J. 2015 Apr 16;20:381. doi: 10.11604/pamj.2015.20.381.5803.

[7] Talle MA, Bonny A, Scholtz W, Chin A, Nel G, Karaye KM, Anzouan-Kacou JB, Damasceno A, Lubenga YR, Sani MU, Mayosi BM. Status of cardiac arrhythmia services in Africa in 2018: a PASCAR Sudden Cardiac Death Task Force report. Cardiovasc J Afr. 2018 Mar/Apr;29(2):115-121. doi: 10.5830/CVJA-2018-027. 
[8] Bonny A, Ngantcha M, Scholtz W, Chin A, Nel G, Anzouan-Kacou JB, Karaye KM, Damasceno A, Crawford TC. Cardiac Arrhythmias in Africa: Epidemiology, Management Challenges, and Perspectives. J Am Coll Cardiol. 2019 Jan 8;73(1):100-109. doi: 10.1016/j.jacc.2018.09.084.

[9] Bonny A, Ngantcha M, Jeilan M, Okello E, Kaviraj B, Talle MA, Nel G, Marijon E, Sani MU, Yousef Z, Karaye KM, Touré IA, Awad MA, Millogo G, Kologo J, Kane A, Houndolo R, Dzudié A, Mbakwem A, Mayosi BM, Chin A. Statistics on the use of cardiac electronic devices and interventional electrophysiological procedures in Africa from 2011 to 2016: report of the Pan African Society of Cardiology (PASCAR) Cardiac Arrhythmias and Pacing Task Forces. Europace. 2018 Sep 1;20(9):1513-1526. doi: 10.1093/europace/eux353.

[10] Worldometer. Perspectivas da população mundial: a revisão de 2019 disponível em: https://www.worldometers.info/populat ion/countries-in-africa-by-population/

[11] Millogo GR, Seghda A, Ilboudo M, Konaté L, Bassolet B, Kologo JK, et al. Five years assessment of cardiac stimulation in two public hospitals in Burkina Faso: An experiment of collaboration with two hospitals in Auvergne. In Annales de cardiologie et d'angeiologie. 2017;66(5):255-259. doi:10.1016/j.ancard.2017.09.018.

[12] Falase B, Sanusi M, Johnson A, Akinrinlola F, Ajayi R, Oke D. Analysis of a five year experience of permanent pacemaker implantation at a Nigerian Teaching Hospital: need for a national database. The Pan African Medical Journal. 2013;16. doi: 10.11604\%2Fpamj.2013.16.16.2644.

[13] Capingana DP, Magalhães $P$, Silva $\mathrm{AB}$, Gonçalves MA, Baldo MP, Rodrigues SL, Simões CC, Ferreira AV, Mill JG. Prevalence of cardiovascular risk factors and socioeconomic level among public-sector workers in Angola. BMC Public Health. 2013 Aug 7;13:732. doi: 10.1186/1471-2458-13-732.

[14] Thomas MO, Oke DA, Ogunleye EO, Adeyanju FA. Bradypacing: indications and management challenges in Nigeria. Pacing Clin Electrophysiol. 2007 Jun;30(6):761-3. doi: 10.1111/j.15408159.2007.00747.x.

[15] Dretzke J, Toff WD, Lip GY, Raftery J, Fry-Smith A, Taylor R. Dual chamber versus single chamber ventricular pacemakers for sick sinus syndrome and atrioventricular block. Cochrane Database Syst Rev. 2004;2004(2):CD003710. doi: 10.1002/14651858.CD003710.pub2.

[16] Klug D, Balde M, Pavin D, HiddenLucet F, Clementy J, Sadoul N, Rey JL, Lande G, Lazarus A, Victor J, Barnay C, Grandbastien B, Kacet S; PEOPLE Study Group. Risk factors related to infections of implanted pacemakers and cardioverter-defibrillators: results of a large prospective study. Circulation. 2007 Sep 18;116(12):1349-55. doi: 10.1161/CIRCULATIONAHA.106.67866 4. 
[17] Thiam M, Fall PD, Gning SB, Ott D, Guèye PM, Wade B, Perret JL. La stimulation cardiaque définitive en Afrique de l'Ouest: faisabilité, problèmes et perspectives [Cardiac pacing in West Africa: feasibility, problems, and perspectives]. Ann Cardiol Angeiol (Paris). 2003 Aug;52(4):212-4. French. doi: 10.1016/s0003-3928(02)00189-0.

[18] Falase B, Sanusi M, Johnson A, Akinrinlola F, Ajayi R, Oke D. Analysis of a five year experience of permanent pacemaker implantation at a Nigerian Teaching Hospital: need for a national database. Pan Afr Med J. 2013 Sep 15;16:16.

doi: 10.11604/pamj.2013.16.16.2644.

[19] Ochasi A, Clark P. Reuse Of Pacemakers In Ghana And Nigeria: Medical, Legal, Cultural And Ethical Perspectives. Dev World Bioeth. 2015 Dec;15(3):125-33. doi: 10.1111/dewb.12047.

[20] Santos F, Rubio E, Abreu L, Barouche E, Mateos J, Mateos E, Mateos J. Cardiac pacemaker: when to indicate and how to use it. Journal of the Faculty of Medical Sciences of Sorocaba. 2008;10(4):5-7.

Conflict of interest: Os autores declaram não haver conflito de interesses com a pesquisa.

Acknowledgements: Aos Drs. Roger Ravelo Dopico e Feliciano Chanana Paquissi. Aos Enfermeiros Guillermo Prades, Kassefo Inglês e Gelson Pereira.
Funding: Nenhum.

How to cite this article: Vicente MBA, Ricardo JRB, Baião D, Manuel EMMR, Martins T, Cordeiro LB, Sabola P, Filipe Júnior AP. Caracterização clínicoepidemiológica dos pacientes submetidos a implantação de dispositivos eletrônicos cardíacos em um hospital terciário privado Angolano. Brazilian Journal of Case Reports. 2021Jul-Sep;01(3):143-151. 